\title{
BMJ Global Health Severity and burden of hand, foot and mouth disease in Asia: a modelling study
}

\author{
Wee Ming Koh, ${ }^{1}$ Hishamuddin Badaruddin, ${ }^{2}$ Hanh La, ${ }^{1}$ Mark I-Cheng Chen, ${ }^{1,3}$ \\ Alex R Cook ${ }^{1}$
}

To cite: Koh WM, Badaruddin $\mathrm{H}$ La $\mathrm{H}$, et al. Severity and burden of hand, foot and mouth disease in Asia: a modelling study. BMJ Glob Health 2018:3:e000442. doi:10.1136/ bmjgh-2017-000442

Handling editor Seye Abimbola

- Additional material is published online only. To view please visit the journal online (http://dx.doi.org/10.1136/ bmjgh-2017-000442).

Received 15 June 2017 Revised 8 October 2017 Accepted 11 October 2017

\section{Check for updates}

${ }^{1}$ Saw Swee Hock School of Public Health, National University of Singapore and National University Health System, Singapore

${ }^{2}$ Ministry of Health, Singapore

${ }^{3}$ Communicable Disease

Centre, Tan Tock Seng Hospital, Singapore

Correspondence to

Dr Alex R Cook;

alex.richard.cook@gmail.com

\section{ABSTRACT}

Background Hand, foot and mouth disease (HFMD) affects millions of children across Asia annually, leading to an increase in implemented control policies such as surveillance, isolation and social distancing in affected jurisdictions. However, limited knowledge of disease burden and severity causes difficulty in policy optimisation as the associated economic cost cannot be easily estimated. We use a data synthesis approach to provide a comprehensive picture of HFMD disease burden, estimating infection risk, symptomatic rates, the risk of complications and death, and overall disability-adjusted life-year (DALY) losses, along with associated uncertainties. Methods Complementary data from a variety of sources were synthesised with mathematical models to obtain estimates of severity of HFMD. This includes serological and other data extracted through a systematic review of HFMD epidemiology previously published by the authors, and laboratory investigations and sentinel reports from Singapore's surveillance system.

Results HFMD is estimated to cause $96900(95 \% \mathrm{Cl}$ 40600 to 259000) age-weighted DALYs per annum in eight high-burden countries in East and Southeast Asia, with the majority of DALYs attributed to years of life lost. The symptomatic case hospitalisation rate of HFMD is $6 \%(2.8 \%-14.9 \%)$, of which $18.7 \%(6.7 \%-31.5 \%)$ are expected to develop complications. $5 \%(2.9 \%-7.4 \%)$ of such cases are fatal, bringing the overall case fatality ratio to be 52.3 (24.4-92.7) per 100000 symptomatic infections. In contrast, the EV-A71 case fatality ratio is estimated to be at least $229.7(75.4-672.1)$ per 100000 symptomatic cases. Asymptomatic rate for EV-A71 is $71.4 \%(68.3 \%-74.3 \%)$ for ages $1-4$, the years of greatest incidence.

Conclusion Despite the high incidence rate of HFMD, total DALY due to HFMD is limited in comparison to other endemic diseases in the region, such as dengue and upper respiratory tract infection. With the majority of DALY caused by years of life lost, it is possible to mitigate most with increased EV-A71 vaccine coverage.

\section{INTRODUCTION}

Hand, foot and mouth disease (HFMD) is a paediatric disease which, although usually self-limiting, can result in complications associated with the central nervous system (CNS)

\section{Key questions}

What is already known about this topic?

- Hand, foot and mouth disease (HFMD) is a highincidence paediatric disease with notified cases exceeding a million each year. Although the number of mild cases vastly outnumbers severe cases, the potential for complications and death has led to an inflated perception of disease severity, which incited a stronger response than necessary in multiple jurisdictions in Asia.

- Although the symptomatic case fatality rate (sCFR) of HFMD has been computed in the literature, it is a poor reflection of the general severity and disease burden because it is sensitive to the notification rates of the surveillance system.

\section{What are the new findings?}

- Using a combination of Singapore's surveillance data along with serological and epidemiological data found in the literature, we constructed a series of models and indices that describe the entire spectrum of HFMD severity. These findings were translated into an age-weighted disability-adjusted life-years (DALY) for comparison to other diseases.

- Our results show that after correcting for non-notification sCFR is likely to be inflated many times. Also, disability plays a minor part in overall DALY and the majority of DALYs could be lowered if fatality rate is lowered, possibly with the EV-A71 vaccine. High asymptomatic rates have profound implications to the modelling studies previously conducted to estimate the reproduction number of EV-A71, which have been generally heterogeneous.

Recommendations for policy

- Policies directed at HFMD should be re-evaluated based on this updated estimate of disease severity and burden.

or death. ${ }^{12}$ The viruses that cause HFMD are particularly widespread in Asia, where they are responsible for millions of infections each year. ${ }^{3}$ An outbreak in Malaysia between April and June 1997 in which 29 children $\operatorname{died}^{4}$ was a harbinger of fatal outbreaks that 
have continued to recent years (170 deaths in Vietnam in $2011^{5}$ and 98 deaths in Cambodia in 2012). ${ }^{6}$ These fatal outbreaks have influenced the perceived severity of HFMD and led to the demand for control measures, ${ }^{7}$ such as the recent phase III vaccine trials in China, ${ }^{8}$ despite the limited information on the disease burden. Many affected countries, such as China, Japan and Singapore, adopted routine control measures akin to those from pandemic preparedness plans-including surveillance, mandatory reporting, isolation, school closures and social distancing-but optimal use of such interventions requires the disease burden of HFMD to be quantified so that the public health response can be calibrated accordingly.

The main aetiological agents of HFMD are the human enterovirus A71 (EV-A71) and coxsackieviruses 10 and 16, with EV-A71 being associated with more severe outcomes. As with other usually mild diseases where infections vastly exceed severe cases, efforts to track all HFMD infections to determine indices for different levels of severity are impractical due to the large number of cases involved. ${ }^{9}$ With the exception of a few jurisdictions, much of the data on HFMD are based on subpopulations, such as the catchment of a health system, a network of sentinel clinics or from passive surveillance using symptomatic case notifications (for instance, ref 10). Such studies may provide an incomplete view of the true burden of disease. Obtaining a more comprehensive picture requires synthesising available information from studies across all severity levels, from serological studies that estimate infection risk, to notification data that provide information on symptomatic cases that could be clinically identified, to hospital records that showcase the risk of more severe expressions such as CNS complications or death. Such estimates need to be constructed carefully to allow uncertainty to be properly reflected and accounted for. This can be achieved through Bayesian methods which provide a flexible framework to integrate data from multiple sources and have been used to estimate infection rates and severity indices for influenza ${ }^{1112}$ and HIV. ${ }^{13}$

To quantify the extent of HFMD across Asia, its mortality risk and the economic burden of control measures against it, ${ }^{7}$ we conducted an analysis using data from a previous systematic review of research across East and Southeast Asia ${ }^{14}$ to develop estimates of disease burden at different levels of severity. Specifically, we adopted a modelling approach that combined serological surveys, epidemiological studies and surveillance data to estimate the risk of children being infected, symptomatic, hospitalised, afflicted with complications and dying. These estimates and the age-weighted method ${ }^{15}$ were used to derive estimates of disability-adjusted life-years (DALY) lost across Asia due to HFMD.

\section{METHODS}

The severity of infection was classified into tiers which require different levels of care: (i) clinically apparent, or symptomatic, cases; (ii) hospitalisations; (iii) complications, or severe cases when the child displays neurological or cardiopulmonary complications; and (iv) fatal cases. These manifestations were matched against similar definitions from the Global Burden of Disease 2013 (GBD2013) study ${ }^{16}$ that have existing estimates of disability weights, and the overall disease burden is the average of each level of severity, weighted by the proportion of cases entering into each level.

These severity tiers were arranged as a pyramid where lower echelons represent the majority of (milder) infections with decreasing frequency but increasing severity towards the apex ${ }^{11}{ }^{17}$ (figure 1). The parameters which describe the severity of HFMD are the proportions of individuals in one state who reach the next higher severity level. This framework implicitly assumes that cases with higher severity are a proportion of cases at the preceding level. For example, those with significant CNS complications are modelled to be a subset of hospitalised cases, and similarly, all hospitalised cases are a subset of notified cases. We built two parallel pyramids for total HFMD (of all aetiologies) and specifically for $E V-A 71$ due to the abundance of data on EV-A71 compared with other viruses, as well as this virus being thought to be responsible for more severe symptoms.

Quantifying the disease burden of HFMD requires an estimate of the total number of cases, which was calculated from the infection rate (both symptomatic and asymptomatic) obtained from seroepidemiological studies. From a systematic review previously published by the authors, ${ }^{14}$ we extracted 10 studies of EV-A71 serology ${ }^{18-28}$ (online supplementary appendix A1, A2 and A3) from which the seropositive rate of EV-A71 by age was found using submodel 1 to model the required infection rate to achieve the observed seroprevalence.

To estimate clinically apparent cases, we used data on notified cases from Singapore, where HFMD is a legally notifiable disease and where active surveillance in preschools is conducted via daily temperature taking; this was used to calculate a lower bound for the overall asymptomatic rate of HFMD, in submodel 2. Indices for higher severity levels of HFMD and EV-A71, which include deaths, complications, hospitalisations and symptomatic cases, used information from 27 papers $^{529-54}$ and 11 papers $^{29} 34353955-61$ respectively from across Asia and were modelled in submodel 3. Finally, the severity indices estimated in the three submodels were fused to collectively estimate the overall disease burden of HFMD in Asia.

\section{Submodel 1: infection rate model}

The infection rate model estimates the age-specific proportion of children who must be infected by the EV-A71 virus each year to reach the observed level of seroprevalence. Serological data from China ${ }^{18-20}$ Singapore, ${ }^{21} 22$ Taiwan, ${ }^{23} 24{ }^{26}$ Thailand $^{27}$ and Vietnam ${ }^{28}$ were extracted from the original papers (figure 2, left), from which it can be observed that seropositivity to EV-A71 starts low at infancy and reaches a plateau around 
Total

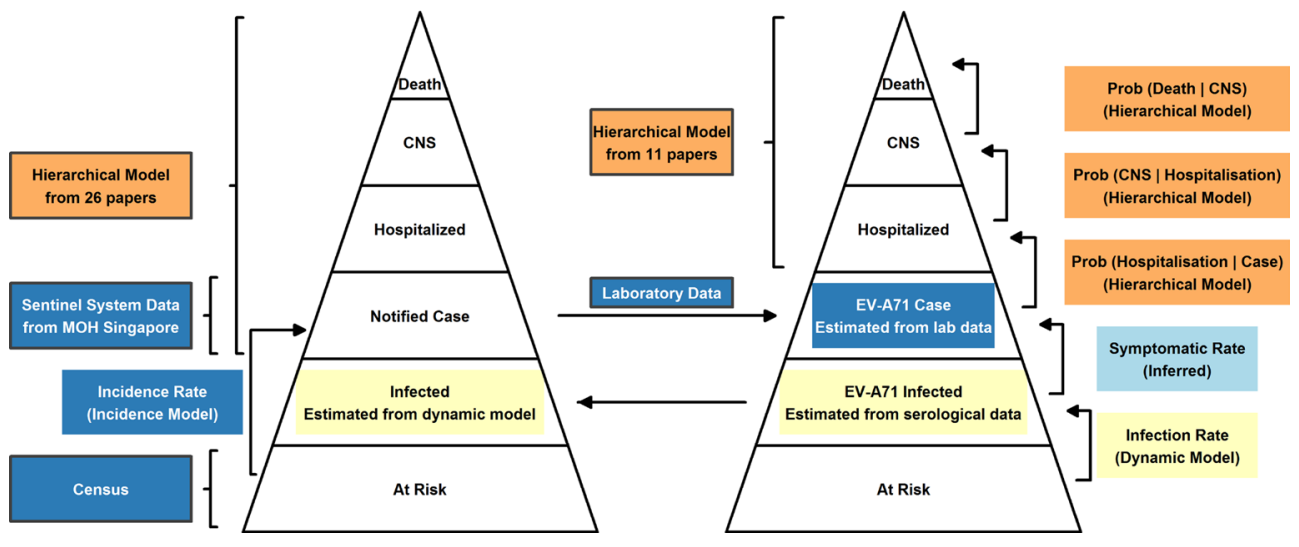

EV-A71 the models are explained further in online supplementary appendix A1.

Posterior distributions of parameters were estimated in $\mathrm{R}^{65}$ using Markov chain Monte Carlo (MCMC) and $\mathrm{R}$ package 'deSolve" ${ }^{66}$ with non-informative prior distributions. The optimal model to represent infection rate was determined by the fit to the data and the model complexity and was evaluated using the deviance information criteria (DIC).

\section{Submodel 2: symptomatic incidence rate model}

Since October 2000, Singapore has adopted a comprehensive approach for HFMD surveillance including a mandatory case reporting system by medical practitioners and childcare centres, ${ }^{67}$ with children at childcares being

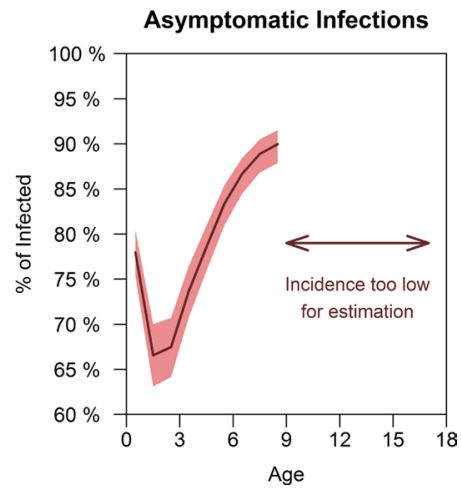

Figure 1 The severity pyramid structure for hand, foot and mouth disease (HFMD) (total, all aetiologies) and EV-A71 in particular. Bordered boxes are data sources, while boxes within the pyramids are estimated quantities. Pale yellow boxes represent the models and outcomes of submodel 1, the infection rate model. This model uses serological data and differential equations to estimate the rate at which infection must occur to match with observed seropositivity level of EV-A71. Blue boxes represent the data, model and outcomes of submodel 2 , the symptomatic incidence rate model. This model uses surveillance data from Singapore to infer the incidence rate of HFMD and EV-A71-specific HFMD. Together with submodel 1, the estimated symptomatic rate of EVA71 (cyan box) can be calculated. The orange boxes represent the data and models of submodel 3: the hierarchical model, which is used to estimate indices that quantifies the higher-level severities of HFMD. CNS, central nervous system; MOH, Ministry of Health.

$40 \%-60 \%$ by adolescence, a finding which is corroborated by cord blood seropositivity (indicative of maternal antibody levels) of $55 \%$ in Vietnam, ${ }^{28} 47 \%-50 \%$ in Taiwan, $^{25} 2638 \%$ in Singapore ${ }^{21}$ and $26 \%$ in China. ${ }^{62}$ To accommodate these patterns, we developed and fit models of the age-specific seroprevalence using seven dynamical models which make varying assumptions for the reason behind these phenomena. These assumptions include (i) an infection rate that drops to zero at age $T$, beyond which no further infection is possible; (ii) an exponentially decreasing infection rate which is asymptotically zero as age increases and (iii) antibodies for EV-A71 in humans will diminish over time like influenza $^{6364}$ and eventually reach an equilibrium from new infections and loss of immunity. The details of each of
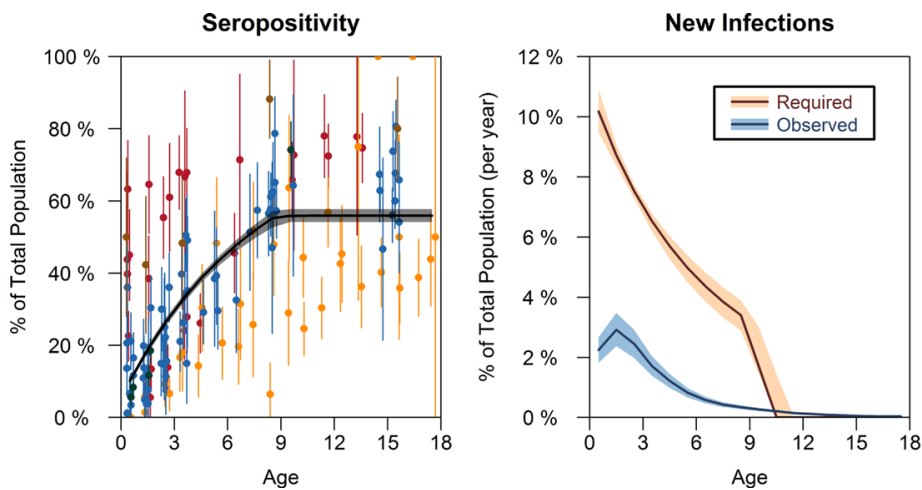

Figure 2 Data and results from the infection rate model (submodel 1). Left: seropositivity levels of EV-A71 with fitted curve. Red data points are from China, blue from Taiwan and orange from Singapore, green from Vietnam and brown from Thailand. The black line is the fitted model with 95\% Bayesian credible intervals. Middle: required and observed rate of EV-A71 infection. The brown line shows the age-specific infection rate of EV-A71 implied from the seropositivity curve and is the required rate to get the observed seropositivity levels; the blue line shows the calculated average EV-A71 infection rate based on Singapore's data from submodel 2. The difference between the two lines are the unobserved cases. Right: asymptomatic infection rate. Calculated from the percentage difference between the required and observed rates of infection in the middle. 

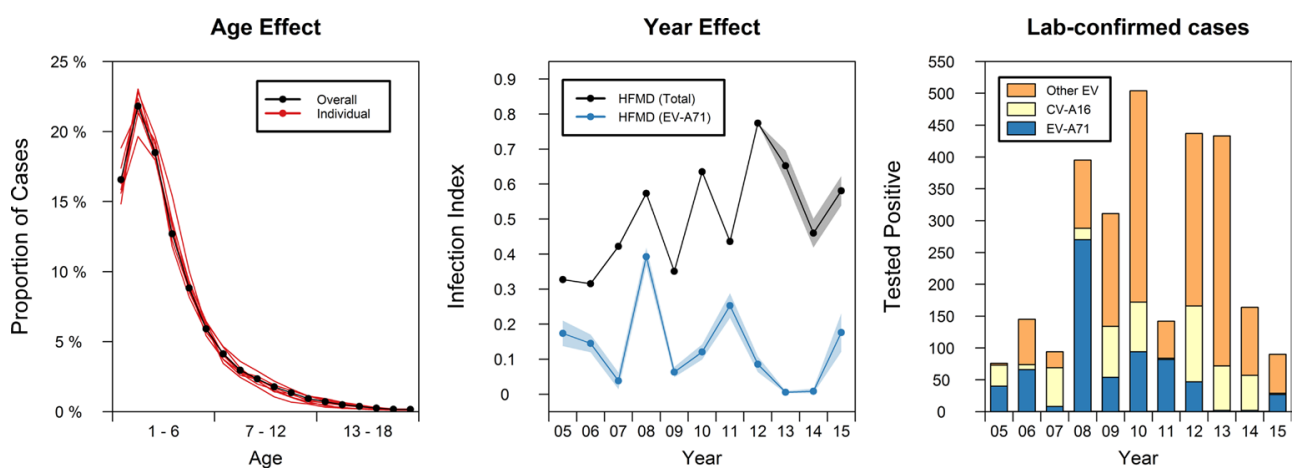

Figure 3 Age and year trend of hand, foot and mouth disease (HFMD) symptomatic infection from the Incidence rate model (submodel 2). Left: the age effect. The black line reflects the overall proportion of incidence for each year of age, and red line represents the age effect for individual years from 2005 to 2012 . Middle: the year effect. The black line is an index which reflects the number of notified cases for 2005-2012. The year effect for 2013-2015 is estimated from data (notified cases) obtained from the Weekly Infectious Disease Bulletin released by the Ministry of Health, Singapore, using linear regression. The year effect of EV-A71 (blue) is mathematically derived from the proportion of cases due to EV-A71 estimated by laboratory data (right). Right: laboratory data from Singapore's surveillance system. This shows the proportion of cases due to each virus in each year from 2005 to 2015.

screened once or twice daily for symptoms and then subsequently isolated for 10 days if symptomatic. The clearly defined population catchment in the city-state and the dual layers of doctor-driven and teacher-driven surveillance make the data on notified symptomatic presentations of HFMD collected by the Singapore's Ministry of Health unusually complete compared with the rest of Asia. In calculating symptomatic incidence rate, we make the assumption that all symptomatic paediatric HFMD were clinically apparent and captured by the surveillance system in Singapore.

We constructed a matrix containing the incidence $m_{a y}$ of cases in year $y$ (2003-2012) among age $a$ (1-18), scaled by the number of individuals in the resident population for that age and year derived from the census. ${ }^{68}$ To separate the effects of age and time on incidence rate, singular value decomposition was performed on the incidence matrix to find the first left-singular vector $u_{a}$ which corresponds to the largest singular value. This vector is normalised to $b_{a}=\frac{u_{a}}{\sum u_{a}}$, which is equivalently the normalised first principal component of $\mathrm{mm}^{\prime}$, and represents the systematic variation attributable to age. The second vector is a time-series index, $\gamma_{y}=\left(u_{a}^{\prime} m\right) \sum u_{a}$, representing the stochastic variation attributable to HFMD incidence level each year. Values for years 2013-2015 were estimated using linear regression using data from Singapore's Weekly Infectious Disease Bulletin. ${ }^{69}$ The modelled HFMD incidence rate is estimated from the product of the two vectors, $\widehat{m}_{a y}=b_{a} \gamma_{y}$.

The EV-A71-specific incidence is a proportion of all HFMD cases. We obtain $\gamma_{y}^{e v}$, the year effect due to EV-A71, by taking it as a binomial sample from the total year effect, $\gamma_{y}^{e v} \sim \operatorname{Bin}\left(\gamma_{y}, p_{y}\right)$. The proportion of cases attributed to EV-A71 in 2008-2015, py, was estimated from data obtained from the National Surveillance Program in Singapore which collects clinical specimens from patients in 107 clinics and 2 hospitals, KK Women's and Children's Hospital and National
University Hospital (figure 3, right). Throat or vesicle swabs were collected from patients $<12$ years of age with clinical diagnosis of HFMD. Specimens were stored at room temperature or $4^{\circ}$ (preferred) for no longer than 48 hours, before being sent to the National Public Health Laboratory for testing and typing. Data on $p$ prior to 2008 were estimated in a study by Ang et al. ${ }^{52}$ The average EV-A71 incidence rate by age is the average of the EV-A71 year effect, weighted by the age effect, or $b_{a} E\left(\gamma_{y}^{e v}\right)$. The model is described in detail in online supplementary appendix B.

\section{Submodel 3: hierarchical model of disease severity}

The upper levels of the severity pyramid from across Asia were modelled as nested binomial distributions, with the probability of reaching state $i$ or higher given the case reaches the lower state $j$ denoted as $\alpha_{i \mid j}$. The states are $D$ (death), $S$ (severe disease, 'CNS'), $H$ (hospitalised) and $C$ (symptomatic case) so that, for example, $\alpha_{H \mid C}$ denotes the case hospitalisation rate. Standard rules of conditional probability mean that the product of these probabilities gives the risk of progressing through multiple levels; for instance, the overall case fatality rate (CFR) is $\alpha_{D \mid C}=\alpha_{D \mid S} \times \alpha_{S \mid H} \times \alpha_{H \mid C}$. As a result, the likelihood function for data with missing tiers of the pyramid is specified via combinations of the basic parameters. For example, 4625 hospitalisations and 11 deaths were documented in the Sarawak 1997 outbreak, ${ }^{49}$ with no mention of severe cases. This data set will allow us to estimate $\pi_{D \mid H}$, and thus make inferences on $\alpha_{D \mid S}$ and $\alpha_{S \mid H}$ through the multiplicative relationship, where $\pi_{i \mid j, p}$ is the individual-level estimate for paper $p$. Detailed description of the model and all data sources can be found in online supplementary appendix C1 (data), C2 and C3 (results) and C4 (model). The posterior distribution of parameters were estimated using the Metropolis Hastings algorithm (MCMC) in R. 


\section{Disability-adjusted life-year burden}

To estimate the DALY lost across Asia to HFMD, we conservatively estimated the incidence of HFMD infection to be twice the incidence of EV-A71 using the incidence rate model (submodel 2), to allow for infection by other aetiological agents of HFMD which tend to be more common than EV-A71. The number of symptomatic cases was then calculated using the asymptomatic rate from the infection rate model (submodel 1). The numbers of more severe manifestations (hospitalised,

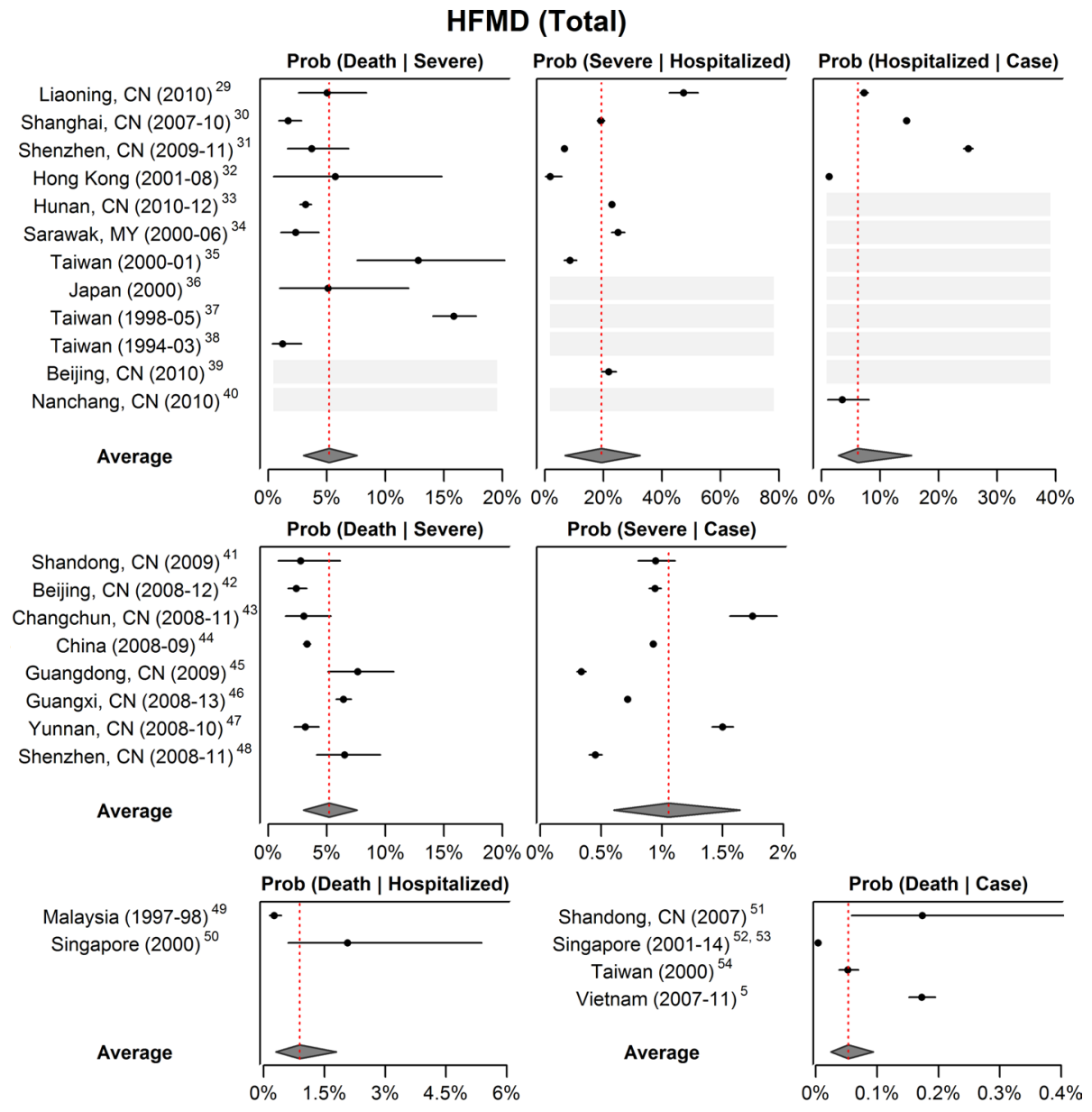

HFMD (EV-A71)

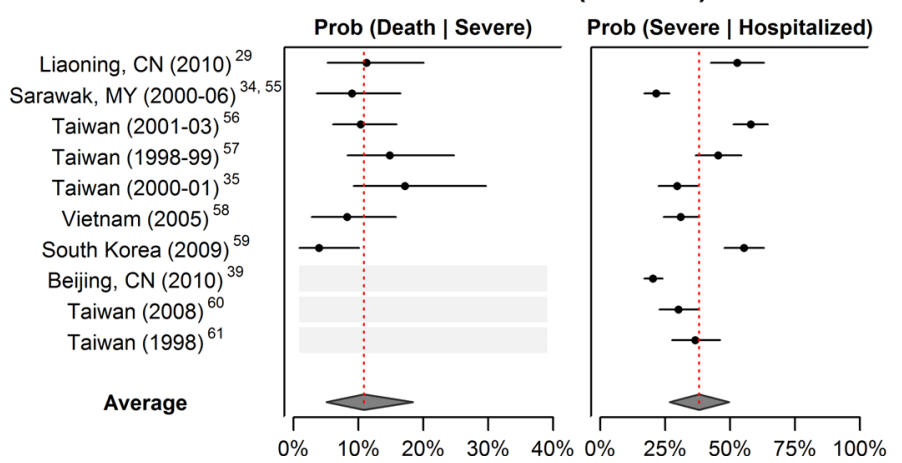

Figure 4 Data and results from the hierarchical model (submodel 3). Top: hand, foot and mouth disease (HFMD) severity for total HFMD. Bottom: HFMD severity for lab-tested EV-A71 cases. These were modelled in two separate hierarchical pyramid models. The median and $95 \%$ credible intervals for each individual paper are shown by the black dot and lines, respectively. All estimates at the individual level are based from a hyperparameter or a higher-level parameter. The vertices of the diamond show the median (also the red line) and 95\% Bayesian credible Intervals. These represent the overall estimate for each severity index, where the estimates are linked together with a multiplicative relationship: $P(D \mid C)=P(D \mid S) \times P(S \mid H) \times P(H \mid C)$. All parameters were estimated using a Bayesian hierarchical approach using Markov chain Monte Carlo. Case hospitalisation ratio estimate for EV-A71 cannot be calculated as studies on EV-A71 cases are almost performed exclusively from hospital data, and thus the lowest level is 'hospitalised' and not 'cases'. 
Table 1 Overall disease severity of hand, foot and mouth disease (HFMD)

\begin{tabular}{|c|c|c|c|c|c|c|}
\hline & HFMD & & Combined & & EV-A71 & \\
\hline Event & Rate & $95 \% \mathrm{Cl}$ & Rate & $95 \% \mathrm{Cl}$ & Rate & $95 \% \mathrm{Cl}$ \\
\hline \multicolumn{7}{|l|}{ Mortality (per 1000 CNS) } \\
\hline $\operatorname{Pr}($ Death | CNS) & 50.2 & (29.2 to 73.5$)$ & - & - & 104.7 & (49.0 to 177.8$)$ \\
\hline \multicolumn{7}{|l|}{ CNS (per 1000 hospitalisations) } \\
\hline $\operatorname{Pr}(\mathrm{CNS} \mid$ Hospitalisation) & 186.7 & (66.6 to 314.9$)$ & - & - & 368.8 & (258. to 480.0$)$ \\
\hline \multicolumn{7}{|l|}{ Hospitalisation (per 1000 cases) } \\
\hline Pr(Hospitalisation | Case) & - & - & 60.2 & (28.2 to 148.6$)$ & - & - \\
\hline \multirow{2}{*}{ Mortality (per 1000 hospitalisations) } & \multicolumn{6}{|c|}{ Product of mortality per CNS and CNS per hospitalisation } \\
\hline & 8.8 & \multicolumn{3}{|c|}{$(2.9$ to 17.7$)$} & 38.3 & (16.6 to 69.5$)$ \\
\hline \multirow[t]{2}{*}{ Mortality (per 100000 cases) } & \multicolumn{6}{|c|}{ Product of mortality per hospitalisation and hospitalisation per case } \\
\hline & 52.3 & $(24.4$ to 92.7$)$ & & & 230.1 & (74.9 to 670.1$)$ \\
\hline \multicolumn{7}{|l|}{ Symptoms (per 1000 infections) } \\
\hline \multicolumn{7}{|l|}{$\operatorname{Pr}($ Case | Infection) } \\
\hline Age 1 & - & - & 220.6 & (195.8 to 247.7$)$ & - & - \\
\hline Age 2 & - & - & 334.6 & (299.7 to 369.7$)$ & - & - \\
\hline Age 3 & - & - & 325.4 & (293.8 to 357.6$)$ & - & - \\
\hline Age 4 & - & - & 263.1 & (236 to 291.5$)$ & - & - \\
\hline Age 5 & - & - & 214.0 & (189.8 to 240.6$)$ & - & - \\
\hline Age 6 & - & - & 166.6 & (146.6 to 191$)$ & - & - \\
\hline Age 7 & - & - & 133.0 & (115.3 to 154.8$)$ & - & - \\
\hline Age 8 & - & - & 111.2 & (95.1 to 131.9$)$ & - & \\
\hline Age 9 & - & - & 100.3 & (84.8 to 121.2$)$ & - & - \\
\hline \multicolumn{7}{|l|}{ Infection (per 1000 person-years) } \\
\hline \multicolumn{7}{|l|}{$\operatorname{Pr}(\operatorname{Infection})$} \\
\hline Age 1 & 44.9 & (36.4 to 53.4$)$ & - & - & 22.4 & (18.2 to 26.7 ) \\
\hline Age 2 & 58.4 & (47.4 to 69.5$)$ & - & - & 29.2 & (23.7 to 34.7 ) \\
\hline Age 3 & 49.0 & (39.8 to 58.3 ) & - & - & 24.5 & (19.9 to 29.1 ) \\
\hline Age 4 & 34.3 & (27.9 to 40.8 ) & - & - & 17.2 & (13.9 to 20.4) \\
\hline Age 5 & 24.3 & (19.8 to 28.9 ) & - & - & 12.2 & (9.9 to 14.5$)$ \\
\hline Age 6 & 16.5 & (13.4 to 19.7 ) & - & - & 8.3 & (6.7 to 9.8 ) \\
\hline Age 7 & 11.6 & (9.4 to 13.8 ) & - & - & 5.8 & (4.7 to 6.9 ) \\
\hline Age 8 & 8.5 & (6.9 to 10.2$)$ & - & - & 4.3 & (3.5 to 5.1 ) \\
\hline Age 9 & 6.8 & (5.5 to 8.1 ) & - & - & 3.4 & (2.8 to 4.1 ) \\
\hline
\end{tabular}

CNS, central nervous system.

CNS and deaths) were then estimated using the parameters from the hierarchical model (submodel 3). Again, posterior distributions were used to characterise uncertainty in these estimates.

Disability weights from GBD2013 ${ }^{16}$ for severe $(0.133$, $0.088-0.19)$, moderate $(0.051,0.032-0.074)$ and mild $(0.006,0.002-0.012)$ acute episodes of infectious disease were used as estimates for HFMD with complication, hospitalisation and symptomatic HFMD, respectively. To estimate the disability duration of each tier, we extracted commonly reported summary statistics such as the sample size, mean, median, range, CIs and variances of the disability duration of their samples from 31 papers and modelled them into Weibull distributions which best describe the statistical properties (online supplementary appendix D). The disability duration for fatalities was determined using the period life expectancy of Japan, ${ }^{70}$ which represents the expected lifespan of humans as healthcare improves in the current century.

We used the age-weighted approach ${ }^{15}$ which assigns greater weights to illnesses that occur during periods when the social role is higher, most notably between ages 10 and 55. As disability from HFMD usually occurs among children, this method provides a conservative estimate of DALY. 
Table 2 Annual disability-adjusted life-year (DALY) losses in eight Asian countries/regions with $95 \%$ credible intervals (Cl)

\begin{tabular}{|c|c|c|}
\hline Country or region & DALY & $95 \% \mathrm{Cl}$ \\
\hline $\begin{array}{l}\text { People's Republic of China } \\
\text { (excluding Hong Kong and } \\
\text { Taiwan) }\end{array}$ & 75881 & (31 835 to 202591 ) \\
\hline $\begin{array}{l}\text { Hong Kong special } \\
\text { administrative region, } \\
\text { People's Republic of China }\end{array}$ & 285 & (115 to 767$)$ \\
\hline Japan & 5456 & (2290 to 14589 ) \\
\hline Malaysia & 2723 & (1138 to 7281$)$ \\
\hline Singapore & 259 & (104 to 748$)$ \\
\hline Taiwan, Republic of China & 1084 & (435 to 3052 ) \\
\hline Thailand & 3928 & (1644 to 10536$)$ \\
\hline Vietnam & 7248 & (3042 to 19414 ) \\
\hline
\end{tabular}

Under this framework, the formula for DALY for an individual is

$$
D A L Y=-\left[\frac{D C e^{-\beta a}}{(\beta+r)^{2}}\left[e^{-(\beta+r) L}(1+((\beta+r)(L+a))-(1+(\beta+r) a)]\right]\right.
$$

where $D$ is the disability weight ( 1 for premature mortality), $r=0.03$ is the discount rate, $C=0.166$ is the age-weighting correcting coefficient, $\beta=0.04$ is the parameter from the age-weighting function, $a$ is the age of onset and $L$ is the duration of disability or time lost due to premature mortality.

\section{RESULTS}

\section{Submodel 1: serological evidence for EV-A71 infection rate}

Among the seven contesting models, model 7 with an unconstrained initial rate of infection and a decreasing rate of infection that reaches 0 at time $T$ has the best fit to the data. The model formulation is

$$
\begin{aligned}
\frac{d I_{t}}{d t} & =c\left(1-I_{t}\right) \alpha_{t} \\
\frac{d \alpha_{t}}{d t} & =-k \alpha_{t} \\
I_{t} & =0, \text { for } t \geq T
\end{aligned}
$$

where $I_{t}$ represents the seropositive rate at time $t, c$ and $k$ are positive real numbers and $\alpha_{t}$ is the rate which seropositivity increases (due to new infections).

The fitted values are $c=2.3$ (95\% CI 1.47 to 5.28$), k=$ $4.79 \%$ (95\% CI $1.92 \%$ to $8.07 \%), T=9$ years $(95 \%$ CI 9 to 10$)$, which translates to around $10 \%$ required infection rate in the first year of life, decreasing to $5 \%$ at ages 5-6, and drops to infinitesimally near $0 \%$ at around age 9 (figure 2, mid). Details of all models, their estimated parameters and the DIC can be found in online supplementary appendix A1, A2 and A3.

\section{Submodel 2: incidence analysis within Singapore}

The year effect, $\gamma_{y}$, which captures the yearly variation of total HFMD incidence (figure 3, mid) has a correlation $>99 \%$ with the overall number of cases of HFMD in Singapore. There is a positive slope of gradient $0.026(95 \%$
CI 0.0003 to 0.052 ) indicating an increase in number of cases over time. The year effect of EV-A71 is estimated using laboratory data (figure 3, right) and does not have a significant trend with slope -0.0091 (95\% CI -0.033 to $0.015)$. Year effects are presented in online supplementary appendix table A4.

The age effect $b_{a}$ (online supplementary appendix table A5) which captures the systematic variation of incidence attributable to age is estimated from data from years 2005 to 2012 and is representative of the proportion of total incidence for each age, showing that $>50 \%$ of disease incidence are due to children <4years of age. Around $2.2 \%-2.9 \%$ of the population is affected by EV-A71 each year during their younger ages (1-3), but this quickly reduces to $1.2 \%$ by age 5 (figure 2 , mid).

For Singapore, the required rate for new infections is much higher than the actual observed level, and the differences are due to asymptomatic infection. For the most vulnerable age group, around $70 \%$ of EV-A71 cases do not show sufficient symptoms to be identified either at presentation to their preschool or by their doctor (figure 2, right). Given the high intensity of Singapore's notification system, this is arguably a lower bound on how much unnotified infection there is across other Asian countries.

\section{Submodel 3: hierarchical model of disease severity}

For HFMD in general (ie, without reference to the specific viral agent), the symptomatic case hospitalisation ratio is estimated to be $6 \%(2.8 \%-14.9 \%)$. Among hospitalised cases, $18.7 \%(6.7 \%-31.5 \%)$ develop CNS complications, of which $5.0 \%(2.9 \%-7.4 \%)$ were fatal. Overall, the CFR for HFMD is estimated to be 52.3 (24.4-92.7) per 100000 symptomatic infections. Among the papers which identified EV-A71 as the pathogen, the majority recruited children seeking treatment in hospitals. Of these, $36.9 \%$ $(25.9 \%-48 \%)$ were associated with CNS complications, of which $10.5 \%(4.9 \%-17.8 \%)$ died. In contrast, there were no discernible differences in the fatality rate of HFMD patients with CNS complications between cases with EV-A71 and all HFMD infections, possibly because most severe cases were indeed caused by EV-A71. Estimates within studies and overall are presented in figure 4 and table 1, which show a summary of all estimated severity quantities of HFMD in Asia with their associated uncertainties.

\section{Disease burden}

The disease burden estimated using our approach is 96900 (40 600-259 000) DALYs per annum in eight high-endemic countries in East and Southeast Asia (estimates by country are provided in table 2). Our approach is conservative and will represent the minimum expected burden of disease. Despite the low CFR, the majority of disability comes from mortality $(\sim 99 \%)$ because deaths from those aged 1 to 3 years contribute very long disability lengths. In contrast, the disability length for mild symptomatic HFMD is estimated at 6.0 (5.8-6.2) days; 
hospitalisation about $8.3(8.1-8.5)$ days, while HFMD with complications at (10.4-11.1) days.

\section{Discussion}

In this study, we used a statistical modelling approach to synthesise information on HFMD from countries in East and Southeast Asia where the disease is highly prevalent. To estimate a conservative lower bound for the asymptomatic rate of EV-A71, we used case notification data from Singapore. The small, clearly demarcated jurisdiction with policies that lead to comprehensive case finding of symptomatic HFMD cases-notification being currently mandatory for physicians and childcare centres-may permit a higher fraction of symptomatic cases to be notified than other settings. Supporting evidence comes from the higher reported incidence rate of HFMD in Singapore (around $6 \%-14 \%$ at age 1 ) than in other areas (eg, about 3-5 times higher than China where the incidence is around $3 \%$ at age $1,{ }^{3}$ despite Singapore having lower average EV-A71 seropositivity rates; figure 2, left). Under the assumption that conditions in Singapore permit all symptomatic HFMD to be detected, then the difference between the infection rate of EV-A71 and the incidence rate of EV-A71 gives an asymptomatic rate which, at $71.4 \%$ $(68.3 \%-74.3 \%)$ for ages $1-4$ (figure 2$)$, is comparable to previous estimates. ${ }^{14}$

Such a high asymptomatic rate for HFMD has implications on estimates of $\mathbf{R}_{0}$, the basic reproduction number. A large proportion of unobserved infections imparts a downward bias on estimated infection rate parameters in compartmental models (such as susceptible-infected-removed variants) that assume all cases are symptomatic, and accounting for the high asymptomatic rate could be the key to reconciling the highly heterogeneous $\mathrm{R}_{0}$ estimates in the literature, which vary from almost unity to over $20 .^{14} 71-75$ Other assumptions that may not always be justified include the assumption that the entire population of a school are susceptible before each outbreak..$^{71} 76$

When performing data synthesis, there is a particular challenge in the allocation of collected information into the severity tiers defined in this study. For instance, we were concerned that the identified EV-A71 cases in the literature were subject to selection bias as it is more likely for physicians to take samples from sicker children. We therefore excluded studies that reported clinically diagnosed cases of EV-A71 because of this potential for bias, and as a result, the lowest level on the severity pyramid we could estimate for EV-A71 was 'hospitalisations' rather than 'symptomatic cases'. To obtain the CFR for EV-A71, therefore, required assuming the case hospitalisation rate is the same for EV-A71 and other aetiologies (ie, $6 \%$, $95 \%$ CI $2.8 \%$ to $14.9 \%$, table 1 ). This is a lower bound estimate since EV-A71 is usually more severe than others. Under that assumption, the CFR of EV-A71 infection is at least $0.23 \%(0.075 \%$ to $0.67 \%$, table 1$)$. This estimate is lower by a factor of 7 than the 2015 systematic review of EV-A71 CFR of $1.7 \%$ (1.2\% to $2.4 \%)^{77}$ which we attribute to a difference in denominator, with the Zhao review more in line with our estimated hospitalisation fatality rate of $3.8 \%(1.7 \%$ to $7.0 \%$, table 1$)$. Based on symptomatic CFRs, EV-A71 is less severe than the relatively mild influenza $\mathrm{A}(\mathrm{H} 1 \mathrm{N1}-2009 \mathrm{pdm})$ strain, which had a symptomatic CFR ranged between $0 \%$ and $1.2 \%$ in 50 published studies reviewed in ref 78. Our other estimates, of case hospitalisation, case severity and CFRs of symptomatic HFMD, align with those from an epidemiological analysis of Chinese data, ${ }^{3}$ giving credibility to both sets of estimates.

Despite the high incidence rate of HFMD, the relative scarcity of severe symptoms and the generally short disability durations led to estimated annual DALY losses in eight high-incidence Asian countries of only 96900 (40 500-258 400), considerably below the disability caused by upper respiratory tract infections (603 000) but comparable to dengue (100000 in the same countries) which has low incidence in China. ${ }^{79}$ However, the disability caused by HFMD is mostly associated with years of life lost $(>99 \%)$, driven by long expected remaining lifespan of children in comparison to the mild, temporary disability from acute HFMD symptoms. Overall, the years of life lost due to HFMD, 96322 (39 911-257 613), are higher than dengue 43762 (25 444-76 488), and upper respiratory tract infection 10767 (8 459-13 $636)$, because the majority of the DALY were caused by years lived with disability for the latter two diseases. These findings provide evidence to support decision-making on adoption of the EV-A71 vaccines.

The challenge in calculating the disease burden of HFMD lies in integrating and synthesising available data from decades of research into useful quantities for public health actions. In doing so, many assumptions were made. In particular, we integrated data from serological and epidemiological studies from a mixture of country-level and city-level studies across Asia to develop an overall 'Asian' average which summarises the severity across affected parts of the continent, which differ in economic development and healthcare systems. These differences cannot be easily adjusted using statistical methods due to low amounts of information from each individual country. Differences between and within countries, however, mean that these overall estimates may not apply to local decision-making. Second, the age-specific asymptomatic rate estimates were based on surveillance data from Singapore, which we believe has a notification system for symptomatic infections presenting at primary care or in education that is close to complete, but with room for misdiagnosis. Third, societal impacts—such as the indirect costs that result from caregivers taking time off work or other impacts of control policies ${ }^{80}$-are not measured in our disease burden estimates, and future research should seek to quantify such indirect impacts of HFMD so that the full cost effectiveness of intervention strategies can be evaluated.

Contributors WMK conceptualised the study, developed the analytical strategy, collected data, performed the statistical analysis, interpreted the results and wrote the first draft of the report. HB contributed to obtaining of data and interpretation 
of the results. $\mathrm{HL}$ and $\mathrm{MI}-\mathrm{CC}$ contributed to the analysis and interpretation of results. ARC contributed to the conceptualisation of the study, analytical strategy, interpretation of results, performed critical revisions of the report and secured funding. All authors contributed to revising the paper and approved the final version.

Funding The work was funded by Singapore's Ministry of Health, Health Services Research grant number HSRG12MAY023, Communicable Disease Public Health Research Grant number CDPHRG12NOV021, the Singapore Population Health Improvement Centre, the Centre for Infectious Disease Epidemiology and Research and the President's Graduate Fellowship to WMK.

Competing interests None declared.

Provenance and peer review Not commissioned; externally peer reviewed.

Data sharing statement Requests for data access should be directed to the corresponding author.

Open Access This is an Open Access article distributed in accordance with the Creative Commons Attribution Non Commercial (CC BY-NC 4.0) license, which permits others to distribute, remix, adapt, build upon this work non-commercially, and license their derivative works on different terms, provided the original work is properly cited and the use is non-commercial. See: http://creativecommons.org/ licenses/by-nc/4.0/

(C) Article author(s) (or their employer(s) unless otherwise stated in the text of the article) 2018. All rights reserved. No commercial use is permitted unless otherwise expressly granted.

\section{REFERENCES}

1. Huang MC, Wang SM, Hsu YW, et al. Long-term cognitive and motor deficits after enterovirus 71 brainstem encephalitis in children. Pediatrics 2006;118:e1785-8.

2. Chang LY, Huang LM, Gau SS, et al. Neurodevelopment and cognition in children after enterovirus 71 infection. $N$ Engl J Med 2007;356:1226-34.

3. Xing W, Liao Q, Viboud C, et al. Hand, foot, and mouth disease in China, 2008-12: an epidemiological study. Lancet Infect Dis 2014;14:308-18.

4. Chan LG, Parashar UD, Lye MS, et al. Deaths of children during an outbreak of hand, foot, and mouth disease in sarawak, malaysia: clinical and pathological characteristics of the disease. For the Outbreak Study Group. Clin Infect Dis 2000;31:678-83.

5. Nguyen NT, Pham HV, Hoang CQ, et al. Epidemiological and clinical characteristics of children who died from hand, foot and mouth disease in Vietnam, 2011. BMC Infect Dis 2014;14:341.

6. Duong V, Mey C, Eloit M, et al. Molecular epidemiology of human enterovirus 71 at the origin of an epidemic of fatal hand, foot and mouth disease cases in Cambodia. Emerg Microbes Infect 2016;5:e104.

7. Siegel K, Cook AR, La H. The impact of hand, foot and mouth disease control policies in Singapore: a qualitative analysis of public perceptions. J Public Health Policy 2017:271-87.

8. Zhu F, Xu W, Xia J, et al. Efficacy, safety, and immunogenicity of an enterovirus 71 vaccine in China. N Engl J Med 2014;370:818-28.

9. Lipsitch M, Hayden FG, Cowling BJ, et al. How to maintain surveillance for novel influenza $\mathrm{A} \mathrm{H} 1 \mathrm{~N} 1$ when there are too many cases to count. The Lancet 2009;374:1209-11.

10. Ho M, Chen E-R, Hsu K-H, et al. An epidemic of enterovirus 71 infection in Taiwan. N Engl J Med Overseas Ed 1999;341:929-35.

11. Presanis AM, De Angelis D, Hagy A, et al. The severity of pandemic H1N1 influenza in the United States, from April to July 2009: a Bayesian analysis. PLoS Med 2009;6:e1000207.

12. Lee VJ, Chen MI, Yap J, et al. Comparability of different methods for estimating influenza infection rates over a single epidemic wave. Am $J$ Epidemiol 2011;174:468-78.

13. Goubar A, Ades AE, De Angelis D, et al. Estimates of human immunodeficiency virus prevalence and proportion diagnosed based on Bayesian multiparameter synthesis of surveillance data. J R Stat Soc Ser A Stat Soc 2008;171:541-80.

14. Koh WM, Bogich T, Siegel K, et al. The epidemiology of hand, foot and mouth disease in Asia: a systematic review and analysis. Pediatr Infect Dis J 2016;35:e285-e300.

15. Murray CJ. Quantifying the burden of disease: the technical basis for disability-adjusted life years. Bull World Health Organ 1994;72:429-45.

16. Salomon JA, Haagsma JA, Davis A, et al. Disability weights for the global burden of disease 2013 study. Lancet Glob Health 2015;3:e712-e723.
17. Garske T, Legrand J, Donnelly CA, et al. Assessing the severity of the novel influenza A/H1N1 pandemic. BMJ 2009;339:b2840-224.

18. Li W, Yi L, Su J, et al. Seroprevalence of human enterovirus 71 and coxsackievirus A16 in Guangdong, China, in pre- and post-2010 HFMD epidemic period. PLoS One 2013;8:e80515.

19. $\mathrm{Yu} \mathrm{H}$, Wang $\mathrm{M}$, Chang $\mathrm{H}$, et al. Prevalence of antibodies against enterovirus 71 in children from Lu'an City in Central China. Jpn J Infect Dis 2011;64:528-32.

20. Zeng M, El Khatib NF, Tu S, et al. Seroepidemiology of enterovirus 71 infection prior to the 2011 season in children in Shanghai. J Clin Virol 2012;53:285-9.

21. Ooi EE, Phoon MC, Ishak B, et al. Seroepidemiology of human enterovirus 71, Singapore. Emerg Infect Dis 2002;8:995-7.

22. Ang LW, Phoon MC, Wu Y, et al. The changing seroepidemiology of enterovirus 71 infection among children and adolescents in Singapore. BMC Infect Dis 2011;11:270.

23. Lu CY, Lee CY, Kao CL, et al. Incidence and case-fatality rates resulting from the 1998 enterovirus 71 outbreak in Taiwan. J Med Virol 2002;67:217-23.

24. Chang LY, King CC, Hsu KH, et al. Risk factors of enterovirus 71 infection and associated hand, foot, and mouth disease/herpangina in children during an epidemic in Taiwan. Pediatrics 2002;109:e88.

25. Luo ST, Chiang PS, Chao AS, et al. Enterovirus 71 maternal antibodies in infants, Taiwan. Emerg Infect Dis 2009;15:581-4.

26. Lee MS, Lin TY, Chiang PS, et al. An investigation of epidemic enterovirus 71 infection in Taiwan, 2008: clinical, virologic, and serologic features. Pediatr Infect Dis J 2010;29:1030-4.

27. Linsuwanon P, Puenpa J, Huang SW, et al. Epidemiology and seroepidemiology of human enterovirus 71 among Thai populations. J Biomed Sci 2014;21:16.

28. Tran CB, Nguyen HT, Phan HT, et al. The seroprevalence and seroincidence of enterovirus71 infection in infants and children in $\mathrm{Ho}$ Chi Minh City, Viet Nam. PLoS One 2011;6:e21116.

29. Xu W, Liu CF, Yan L, et al. Distribution of enteroviruses in hospitalized children with hand, foot and mouth disease and relationship between pathogens and nervous system complications. Virol J 2012;9:8.

30. Zeng M, Li YF, Wang XH, et al. Epidemiology of hand, foot, and mouth disease in children in Shanghai 2007-2010. Epidemiol Infect 2012;140:1122-30

31. Wang YR, Sun LL, Xiao WL, et al. Epidemiology and clinical characteristics of hand foot, and mouth disease in a Shenzhen sentinel hospital from 2009 to 2011. BMC Infect Dis 2013;13:539.

32. Ma E, Lam T, Chan KC, et al. Changing epidemiology of hand, foot and mouth disease in Hong Kong, 2001-2009. Jpn J Infect Dis 2010;63:422-6.

33. Gao LD, Hu SX, Zhang $\mathrm{H}$, et al. Correlation analysis of EV71 detection and case severity in hand, foot, and mouth disease in the Hunan Province of China. PLoS One 2014:9:e100003.

34. Ooi MH, Wong SC, Mohan A, et al. Identification and validation of clinical predictors for the risk of neurological involvement in children with hand, foot, and mouth disease in Sarawak. BMC Infect Dis 2009;9:3.

35. Lu HK, Lin TY, Hsia SH, et al. Prognostic implications of myoclonic jerk in children with enterovirus infection. J Microbiol Immunol Infect 2004;37:82

36. Fujimoto T, Chikahira M, Yoshida S, et al. Outbreak of central nervous system disease associated with hand, foot, and mouth disease in Japan during the summer of 2000: detection and molecular epidemiology of enterovirus 71. Microbiol Immunol 2002;46:621-7

37. Chen KT, Chang HL, Wang ST, et al. Epidemiologic features of handfoot-mouth disease and herpangina caused by enterovirus 71 in Taiwan, 1998-2005. Pediatrics 2007;120:e244-e252.

38. Yang TT, Huang LM, Lu CY, et al. Clinical features and factors of unfavorable outcomes for non-polio enterovirus infection of the central nervous system in northern Taiwan, 1994-2003. J Microbiol Immunol Infect 2005;38:417-24.

39. Zhu D, Zhao XY, Yao Y, et al. A new factor influencing pathogen detection by molecular assay in children with both mild and severe hand, foot, and mouth disease. Diagn Microbiol Infect Dis 2013;76:162-7

40. Liu MY, Liu W, Luo J, et al. Characterization of an outbreak of hand, foot, and mouth disease in Nanchang, China in 2010. PLoS One 2011;6:e25287.

41. Tian H, Yang QZ, Liang J, et al. Clinical features and management outcomes of severe hand, foot and mouth disease. Med Princ Pract 2012;21:355-9.

42. Wang J, Cao Z, Zeng DD, et al. Epidemiological analysis, detection, and comparison of space-time patterns of Beijing hand-foot-mouth disease (2008-2012). PLoS One 2014;9:e92745. 
43. Yan L, Li X, Yu Y, et al. Distribution and risk factors of hand, foot, and mouth disease in Changchun, northeastern China. Chin Sci Bull 2014;59:533-8.

44. Wang $Y$, Feng Z, Yang $Y$, et al. Hand, foot, and mouth disease in China: patterns of spread and transmissibility. Epidemiology 2011;22:781-92.

45. De W, Changwen K, Wei L, et al. A large outbreak of hand, foot, and mouth disease caused by EV71 and CAV16 in Guangdong, China, 2009. Arch Virol 2011;156:945-53.

46. Xie YH, Chongsuvivatwong V, Tang Z, et al. Spatio-temporal clustering of hand, foot, and mouth disease at the county level in Guangxi, China. PLoS One 2014;9:e88065.

47. Xu W, Jiang L, Thammawijaya $P$, et al. Hand, foot and mouth disease in Yunnan Province, China, 2008-2010. Asia Pac J Public Health 2015;27:NP769-NP777.

48. Mou J, Dawes M, Li Y, et al. Severe hand, foot and mouth disease in Shenzhen, South China: what matters most? Epidemiol Infect 2014;142:776-88.

49. Shekhar K, Lye MS, Norlijah O, et al. Deaths in children during an outbreak of hand, foot and mouth disease in Peninsular Malaysia--clinical and pathological characteristics. Med J Malaysia 2005;60:297-304.

50. Chong CY, Chan KP, Shah VA, et al. Hand, foot and mouth disease in Singapore: a comparison of fatal and non-fatal cases. Acta Paediatr 2003;92:1163-9.

51. Zhang Y, Tan XJ, Wang HY, et al. An outbreak of hand, foot, and mouth disease associated with subgenotype $\mathrm{C} 4$ of human enterovirus 71 in Shandong, China. J Clin Virol 2009;44:262-7.

52. Ang LW, Koh BK, Chan KP, et al. Epidemiology and control of hand, foot and mouth disease in Singapore, 2001-2007. Ann Acad Med Singapore 2009;38:106-12

53. Wu Y, Yeo A, Phoon MC, et al. The largest outbreak of hand; foot and mouth disease in Singapore in 2008: the role of enterovirus 71 and coxsackievirus A strains. Int $J$ Infect Dis 2010;14:e107 6-e1081.

54. Wang JR, Tuan YC, Tsai HP, et al. Change of major genotype of enterovirus 71 in outbreaks of hand-foot-and-mouth disease in Taiwan between 1998 and 2000. J Clin Microbiol 2002;40:10-15.

55. Ooi MH, Wong SC, Podin Y, et al. Human enterovirus 71 disease in Sarawak, Malaysia: a prospective clinical, virological, and molecular epidemiological study. Clin Infect Dis 2007;44:646-56.

56. Chang LY, Chang IS, Chen WJ, et al. HLA-A33 is associated with susceptibility to enterovirus 71 infection. Pediatrics 2008;122:1271-6.

57. Liu CC, Tseng HW, Wang SM, et al. An outbreak of enterovirus 71 infection in Taiwan, 1998: epidemiologic and clinical manifestations. $J$ Clin Virol 2000;17:23-30.

58. Van Tu P, Thao NTT, Perera D, et al. Epidemiologic and virologic investigation of hand, foot, and mouth disease, southern Vietnam, 2005. Emerg Infect Dis 2007;13:1733-41.

59. Ryu WS, Kang B, Hong J, et al. Enterovirus 71 infection with central nervous system involvement, South Korea. Emerg Infect Dis 2010;16:1764-6.

60. Wang SM, Ho TS, Lin HC, et al. Reemerging of enterovirus 71 in Taiwan: the age impact on disease severity. Eur $J$ Clin Microbiol Infect Dis 2012;31:1219-24.

61. Wang SM, Liu CC, Tseng HW, et al. Clinical spectrum of enterovirus 71 infection in children in southern Taiwan, with an emphasis on neurological complications. Clin Infect Dis 1999;29:184-90.
62. Mao QY, Liao XY, Yu X, et al. Dynamic change of mother-source neutralizing antibodies against enterovirus 71 and coxsackievirus A16 in infants. Chin Med J 2010;123:1679.

63. Horsfall FL, Rickard ER. Neutralizing antibodies in human serum after influenza $A$ : the lack of strain specificity in the immunological response. J Exp Med 1941;74:433-9.

64. Hsu JP, Zhao X, Chen MI, et al. Rate of decline of antibody titers to pandemic influenza $A(\mathrm{H} 1 \mathrm{~N} 1-2009)$ by hemagglutination inhibition and virus microneutralization assays in a cohort of seroconverting adults in Singapore. BMC Infect Dis 2014;14:414.

65. R Core Team. R: A language and environment for statistical computing. R Foundation for Statistical Computing. 2016.

66. Soetaert K, Petzoldt T, Setzer RW. Solving differential equations in $R$ : package deSolve. J Stat Softw 2010;33:1-25.

67. Ministry of Health. Hand, foot \& mouth disease. https://www.moh. gov.sg/content/moh_web/home/diseases_and_conditions/h/hfmd. html (accessed 3 May 2016).

68. US Census Bureau. D. I. S. International programs, international data base. https://www.census.gov/population/international/data/idb/ informationGateway.php (accessed 1 Nov 2016).

69. Ministry of Health, Singapore. Weekly infectious diseases bulletin. $\mathrm{h}$ ttps://www.moh.gov.sg/content/moh_web/home/statistics/infectious DiseasesStatistics/weekly infectiousdiseasesbulletin.html (accessed 2 Feb 2017)

70. Ministry of Health, Labour and Welfare, Japan. The 21 th life tables (2010). http://www.mhlw.go.jp/english/database/db-hw/lifetb21th/dl/ tables.pdf (Accessed: 11 Jan 2016)

71. Lim CT, Jiang L, Ma S, et al. Basic reproduction number of coxsackievirus type A6 and A16 and enterovirus 71: estimates from outbreaks of hand, foot and mouth disease in Singapore, a tropical city-state. Epidemiol Infect 2016;144:1028-34.

72. Takahashi S, Liao Q, Van Boeckel TP, et al. Hand, foot, and mouth disease in China: modeling epidemic dynamics of enterovirus serotypes and implications for vaccination. PLoS Med 2016;13:e1001958.

73. Li Y, Zhang J, Zhang X. Modeling and preventive measures of hand, foot and mouth disease (HFMD) in China. Int J Environ Res Public Health 2014;11:3108-17.

74. Li $Y$, Wang $L$, Pang $L$, et al. The data fitting and optimal control of a hand, foot and mouth disease (HFMD) model with stage structure. Appl Math Comput 2016;276:61-74.

75. Yang J-Y, Chen Y, Zhang F-Q. Stability analysis and optimal control of a hand-foot-mouth disease (HFMD) model. J Appl Math Comput 2013;41:99-117.

76. Ma E, Fung $\mathrm{C}$, Yip $\mathrm{SH}$, et al. Estimation of the basic reproduction number of enterovirus 71 and coxsackievirus A16 in hand, foot, and mouth disease outbreaks. Pediatr Infect Dis J 2011;30:675-9.

77. Zhao $Y Y$, Jin $\mathrm{H}$, Zhang XF, et al. Case-fatality of hand, foot and mouth disease associated with EV71: a systematic review and metaanalysis. Epidemiol Infect 2015;143:3094-102.

78. Wong JY, Kelly H, Ip DK, et al. Case fatality risk of influenza A (H1N1pdm09): a systematic review. Epidemiology 2013;24:830-41.

79. Global Burden of Disease Study. Global Burden of Disease Study 2015 (GBD 2015) Results.Seattle, United States: Institute for Health Metrics and Evaluation (IHME) (2016). 2015 http://ghdx.healthdata. org/gbd-results-tool (accessed 6 Feb 2017).

80. Zou S. Applying DALYs to the burden of infectious diseases. Bull World Health Organ 2001;79:267-8. 